\title{
A Probabilistic Approach to Building Change Detection
}

\author{
${ }^{1}$ Abdullah H. Özcan, ${ }^{2}$ Cem Ünsalan, ${ }^{3}$ Peter Reinartz \\ ${ }^{1}$ Tubitak BILGEM, Turkey \\ ${ }^{2}$ Yeditepe University, Turkey \\ ${ }^{3}$ DLR, Germany \\ (e-mail: abdullah.ozcan@tubitak.gov.tr)
}

\begin{abstract}
Among different remote sensing applications, change detection deserves specific consideration. The importance of this area is its applicability on damage assessment after natural disasters. Fortunately, recent sensors allow researchers to develop advanced change detection methods. Some of these benefit from panchromatic or multispectral remote sensing images, whereas others use $3 D$ data besides the $2 D$ information. In this study, we benefit from both $2 \mathrm{D}$ and $3 \mathrm{D}$ data to detect changes in buildings. We specifically focused on building change detection, since after a natural disaster damaged building information is one of the most important one. Our building change detection method is based on our previous study based on probabilistic building detection. In this study, we first extract corner points using the Harris corner detector from panchromatic images. These corner points are used on Digital Surface Model (DSM) data to estimate possible building locations. To do so, we represent possible building locations via a kernel based density estimation method. In this study, we use the difference of the bitemporal estimated kernel maps (obtained in two different times) for change detection. Then, we apply a morphology based shape refinement method. As a result, we can detect changes in the scene. We tested our method on WorldView2 sensor images with 780 buildings. The results are promising.
\end{abstract}

\section{INTRODUCTION}

The importance of change detection in remote sensing is its applicability on damage assessment after natural disasters. Fortunately, recent sensors allow researchers to develop advanced change detection methods. Some of these methods benefit from panchromatic or multispectral remote sensing images, whereas others use $3 \mathrm{D}$ data besides the $2 \mathrm{D}$ information. In this study, we benefit from both $2 \mathrm{D}$ and $3 \mathrm{D}$ data to detect changes on buildings. We specifically focused on building change detection, since after a natural disaster damaged building information is one of the most important one. Our building change detection method is based on our previous study focusing on probabilistic building detection [10]. For more detail on building detection methods, readers can also check our previous study [11].

As mentioned previously, we can group change detection methods into two main categories. In the first category, only $2 \mathrm{D}$ data is used. In these, panchromatic and multispectral data can be used separately or in a joint manner [7]. In the second category, 3D information is used for change detection. The methods in this category also allow detecting depth changes. Change detection using 3D data is extensively studied. Here, we will only mention some benchmark studies. Dini et al. [5] used high resolution stereoscopic satellite images to generate
DSM and detect changes using them. In their method, large differences between two DSMs are used to detect changes. They eliminated some structures that do not belong to actual changes. To do so, they benefit from morphological filtering and GIS database. Tong et al. [15] detected collapsed and damaged buildings by comparing the height of corner points. Sofina and Ehlers [12] used remotely sensed images and vector information for change detection. Jung [8] used DEM and panchromatic images for change detection in a decision tree classification framework. Murakami et al. [9] proposed a change detection method based on LIDAR DSM difference. Besides these, there are also various studies focusing on different feature fusion methods including DSM subtraction of bitemporal data for change detection [1], [2], [3], [13], [14]. In these studies, shape features, classifiers, shadow information, morphological filters, and spectral descriptors are used for pixel or object based change detection. Some of these studies also used different sensor's DSM data.

Although detecting 3D changes using DSM subtraction may seem sufficient, this may not be possible due to several reasons. To name a few, the first reason for this is the automatic DSM generation step. During DSM generation, some unwanted outliers may occur. These are caused by matching errors, temporal changes or applied interpolation techniques. The second reason is the viewing angle of the sensor. The same building may not be represented in exactly the same shape if DSM is constructed with data from different viewing angles. The third reason is the illumination condition and shadows. They may cause unwanted expansion or diminution of generated DSM in the horizontal space. The fourth reason is the used interpolation technique. It may cause loss of sharpness around object boundaries in DSM data. Therefore, buildings may not have clear rooftop contours. The fifth reason is the false object labeling. Sometimes a group of trees may look like a building and there is no easy way to separate them. There are also some cases that DSM subtraction may give misleading results. To give an example assume that there were two buildings. Then they are demolished and one different building is constructed at the same area. Subtracting DSM will not give the correct result in this case.

From the previous paragraph, it should become clear that change detection with only DSM subtraction is not robust. However, the height information in DSM is still very valuable. If the DSM was obtained using LIDAR where the resolution is high, building edges and closely located buildings may be separated. In this setup, DSM subtraction may give very good 
results. However, the generated DSM is still not perfect. If the DSM data is obtained from stereo image pairs, then the change detection results using DSM difference may become less reliable. In Fig. 1, we provide such an example.



Fig. 1. DSM subtraction of bitemporal images. The first column corresponds to panchromatic data. The second column corresponds to DSM data and the third column corresponds to DSM differences.

In this figure, the panchromatic data is given in the first column, DSM data in the second column, and DSM difference on these in the third column. To make the results more readable, we also thresholded DSM differences. As can be seen, some buildings are very close and building edges are not sharp here. Also unchanged buildings don't have the same DSM in the horizontal space due to viewing angle of the sensor and shadows. Therefore, DSM subtraction does not give good results.

To overcome the mentioned problems on DSM based change detection, we use both $2 \mathrm{D}$ and $3 \mathrm{D}$ data in this study. We obtain the 3D data from the stereo pair by the semiglobal matching algorithm [4]. Our building change detection method has four steps. First, we extract corner points using the Harris corner detector from panchromatic images. These corner points are used on DSM data to search for possible building locations. Using a kernel based density estimation method, possible building locations are represented as a kernel density map. Second, the difference of bitemporal kernel density maps obtained from two different times are used for change detection. Third, changed building boundaries are extracted. Fourth, these boundaries are refined using morphological operations. In the following sections, we provide each step in detail.

\section{BuILding Kernel Density Map EXtraction}

In this section, we benefit from our previous work for kernel map extraction with some improvements [10]. For the completeness of this study, we briefly review it here. First, we extract local feature points using the Harris corner detector [6]. Harris corner detector searches intensity changes (in terms of gradient magnitudes) in a local window. This local window is constructed for all pixels in the panchromatic image. Then, corner points are extracted based on the local maxima of intensity changes. Afterwards each corner point, $\left(x_{c}, y_{c}\right)$, is assumed to be in the center of a $w \times w$ window, $I_{s}$, on the DSM. The highest point in this window, $\left(x_{m}, y_{m}\right)$, is obtained based on its elevation data as

$$
\left(x_{m}, y_{m}\right)=\operatorname{argmax}\left(I_{s}(x, y)\right)
$$

where,

$$
\begin{array}{r}
x_{c}-w / 2 \leq x \leq x_{c}+w / 2 \\
y_{c}-w / 2 \leq y \leq y_{c}+w / 2
\end{array}
$$

Then, we take $\left(x_{m}, y_{m}\right)$ as the candidate kernel center. Before assigning a kernel to this location, we apply filtering using the DSM information as follows. If the height difference between the corner point and the maximum height point on $I_{s},\left|I_{s}\left(x_{c}, y_{c}\right)-I_{s}\left(x_{m}, y_{m}\right)\right|$, is less than a threshold, then $\left(x_{m}, y_{m}\right)$ is not considered for kernel formation. Otherwise, we use the symmetric Gaussian probability density function for kernel density estimation as

$$
p(x, y)=\frac{1}{\sqrt{2 \pi} \sigma} \exp \left(-\frac{\left(x-x_{m}\right)^{2}+\left(y-y_{m}\right)^{2}}{2 \sigma^{2}}\right)
$$

where $\sigma=w / 3$ which gives enough space for kernel formation. We apply these steps for all extracted kernel centers, $\left(x_{m}(i), y_{m}(i)\right)$. As a result, we obtain the kernel density map as

$$
p_{\text {map }}(x, y)=\sum_{i=1}^{N} \frac{1}{\sqrt{2 \pi} \sigma} \exp (A(x, y))
$$

where

$$
A(x, y)=-\frac{\left(x-x_{m}(i)\right)^{2}+\left(y-y_{m}(i)\right)^{2}}{2 \sigma^{2}}
$$

In Eqn. 4, $N$ is the number of corner points. As in our previous method, the formed density function can be used to detect building centers in the region. The final density map will be multimodal since the number of buildings is unknown. Local maxima of $p_{\text {map }}(x, y)$ will give possible building locations.

There is one possible weakness of this method. If there are two adjacent buildings with one of them significantly higher than the other, then most of the corner points would indicate the higher building. As a result the lower building will be masked. In order to prevent this, we improve our kernel density estimation method in this study. To do so, we divide the $w \times w$ subwindow into four equal sized windows and search for the maximum height point on each window separately. Therefore, one corner point will indicate at most four points. As a result, high buildings will not mask nearby lower buildings.

We apply the improved kernel density estimation method to the test image in Fig. 1. We provide the results in Fig. 2-a,b. As can be seen in this figure, closely located buildings are well separated in both images. In the next section, we explain our change detection method based on these kernel density maps.

\section{Building Change Detection Using Bitemporal KERNEL DENSITY MAPS}

Based on the derivation in the previous section, we know that the estimated kernel density map for both bitemporal images keep possible building locations. Here, we will use them to detect building changes. To do so, we propose using 

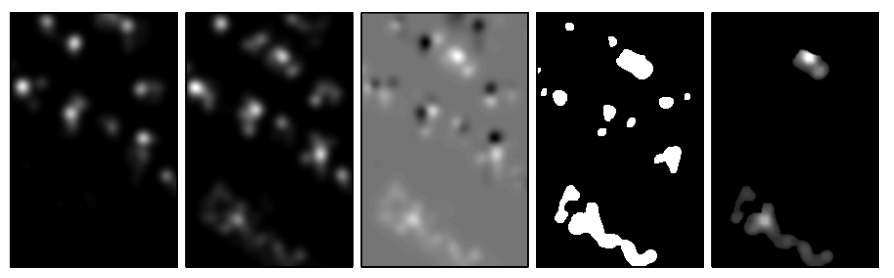

Fig. 2. Summary of the change detection method. The first column corresponds to the extracted kernel map from the first time. The second column corresponds to the extracted kernel map from the second time. The third column corresponds to the difference of kernel maps. The fourth column corresponds to the thresholded difference. The fifth column corresponds to the refined (by DSM difference) changes.

the difference of kernel density maps constructed based on bitemporal images. Since most of kernels are generated around the center of buildings, subtracting kernel density maps will give better results compared to subtracting DSM data in two different times.

In this work, we categorize changes into two categories as follows: a positive change represents a constructed building; a negative change represents a demolished building. For the positive change, the kernel map difference can be obtained as

$$
p_{+}=\left(p_{m a p 2}-p_{m a p 1}\right)>T
$$

Similarly, for the negative change the difference becomes

$$
p_{-}=\left(p_{m a p 1}-p_{m a p 2}\right)>T
$$

where $p_{m a p 1}$ and $p_{\text {map } 2}$ are the kernel density maps obtained from bitemporal images. Here $T$ is the threshold to eliminate some false alarms. During kernel density estimation we set the maximum value of each kernel for each corner point to be one. Based on this setup, we assume that at least three corners should indicate a building. Therefore, we set the threshold $T$ to be three. In actual tests, we expect more than three corners to represent a building. This adds extra robustness for the selected threshold value.

After thresholding, we label each object by connected components analysis. Then, we check each object whether it is changed or not. At this step, we also check the DSM difference on each object. To do so, we take the DSM difference on each pixel in the object. If the mean height difference of the pixels of an object is above a threshold ( 2.5 meter here), then we keep this object for further building boundary extraction.

We apply our building change detection method on the test image set given in Fig. 1. We provide the results in Fig. 2-c. We also provide the thresholded results in Fig.2-d. Here, there are 14 individual objects. There are three changed buildings and we provide their kernel densities in Fig.2-e.

\section{Shape Refinement}

In this section, we extract the changed building footprints and refine their shapes by shadows. To do so, we compute the extended maxima transform of DSM and extract components connected to the object. Then, we apply shadow based edge refinement. We extract shadow areas from panchromatic images using the bimodal histogram splitting method [16]. In the final result we morphologically clean the image. Next, we explain the shape refinement steps in detail.

\section{A. Extended Maxima Transform}

In the first step of shape refinement, we compute the extended maxima transform of the DSM. Extended maxima transform is the regional maxima of the h-maxima transform. The h-maxima transform suppresses all maxima whose depth is below or equal to a threshold $h$ [17]. This is achieved by performing the reconstruction by dilation of DSM from DSM$\mathrm{h}$, which is defined as

$$
\rho_{D S M}(D S M-h)=\underset{n \geqslant 1}{\vee} \delta_{D S M}^{(n)}(D S M-h)
$$

where the operand $\vee$ denotes point-wise maximum operation and $\delta_{D S M}^{(n)}$ denotes the $\mathrm{n}$-fold dilation. Stopping point $n$ is reached when the height value of a pixel in the dilated image is equal (in height) to the pixel in the original image. Then, we extract the regional maxima of $\rho_{D S M}(D S M-h)$ in Eqn. 8 . The result for our test image is given in Fig. 3-a. Here $h$ is chosen as 2.5 meters.

\section{B. Shadow Information}

In the second step of shape refinement, we use the shadow information. Here, we aim to refine the edges and separate closely located buildings. Since buildings are very closely located, we use shadow information to separate them. We extract the shadow areas using bimodal histogram splitting method. Then, we discard these from the final change map. The refined edges are given in Fig. 3-b. Finally, we use morphological opening and fill in the holes respectively to clean the image. We provide the results of this operation in Fig. 3-c.

We extract the final changed buildings using absolute kernel density map differences as shown in Fig. 2-e. Here, we only select buildings from Fig. 3-c that overlap with kernel map differences as given in Fig. 3-d. In the next section, we test our method on different data sets.

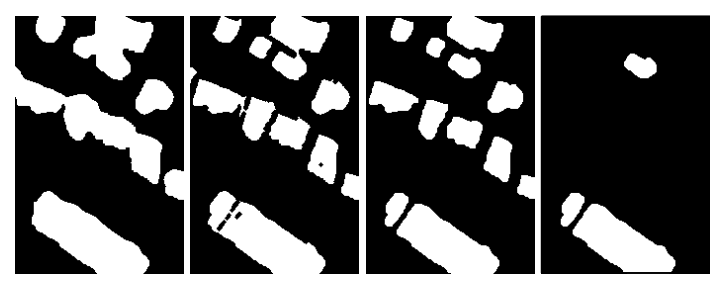

Fig. 3. Shape Refinement. The first column corresponds to extended maxima transform. The second column corresponds to shadow based edge refinement. The third column corresponds to the cleaned image. The fourth column corresponds to changed buildings.

\section{EXPERIMENTS}

In this section, we analyze the building change detection performance of our method. Our test set is based on bitemporal WorldView-2 images containing 780 buildings. In our tests, 
we only detect changes if a new building is constructed or an existing building is demolished. Based on this definition, we provide the building change detection results for each bitemporal image set separately in Table I. In this table, we provide the actual number of changes, true detections, and false alarms.

TABLE I. CHANGE DETECTION PERFORMANCE OF OUR METHOD.

\begin{tabular}{lccc}
\hline Image Set & Total Changes & True Detections & False Alarms \\
\hline Istanbul $_{1}$ & 7 & 7 & 1 \\
Istanbul $_{2}$ & 10 & 9 & 2 \\
Istanbul $_{3}$ & 7 & 6 & 1 \\
Istanbul $_{4}$ & 7 & 6 & 2 \\
\hline Total & 31 & 28 & 6 \\
\hline
\end{tabular}

As can be seen in Table I, there are a total of 31 changes in our test set. Our method was able to detect 28 of them correctly. There are also six false alarms. Missed change detections are mainly caused by low number of corner points. This led to insufficient kernel formation. Three of the false alarms are caused by tree clusters. If we were able to use the multispectral information (such as Normalized Difference Vegetation Index), these false alarms could have been eliminated easily. Two of the false alarms are arisen from DSM generation. In these two cases, DSM of the buildings were not generated in one of the bitemporal images. Hence we had false alarms. The final false alarm is caused by very different DSM information of the same building in two images. This may be because of the viewing angle of the sensor.

In Fig. 4, we provide the detected changes for all our bitemporal image set. The reader may check the change detection performance of our method visually by evaluating this figure.

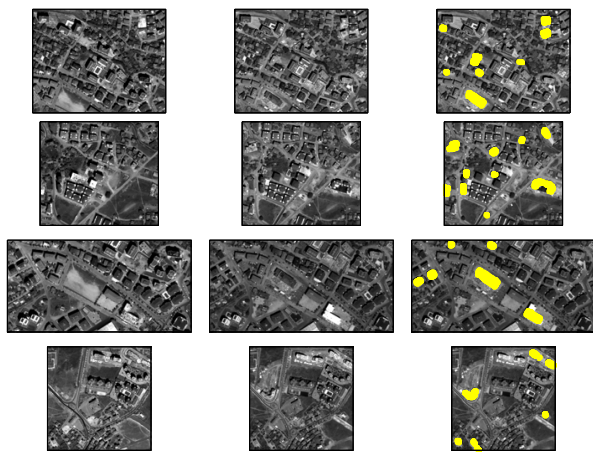

Fig. 4. Change detection results on four bitemporal Istanbul images. First column corresponds to time- 1 , the second column corresponds to time- 2 , the third column corresponds to the categorized changes.

\section{FinAl COMMENTS}

In this study, we proposed a new method for change detection using bitemporal panchromatic images and DSM data. First, we extract corner points using the Harris corner detector on the panchromatic image set. Then, we use these corner points on DSM data to search for possible building locations. Using a kernel based density estimation method, we were able to obtain possible building locations in terms of a kernel density map. This step was for building detection in each image obtained from different times. Then, we used the difference of the kernel density maps (obtained from bitemporal images) to detect constructed and demolished buildings. We tested our method on WorldView-2 sensor images on a 780 building set. Test results on four bitemporal images show the effectiveness of our method.

\section{REFERENCES}

[1] H. Chaabouni, P. dAngelo, T. Krauss, and P. Reinartz, "Automatic urban area monitoring using digital surface models and shape features," JURSE 2011-Joint Urban Remote Sensing Event, 2011.

[2] H. Chaabouni, T. Krauss, P. dAngelo, and P. Reinartz, " $3 \mathrm{~d}$ change detection inside urban areas using different digital surface models," ISPRS PCV 2010-Photogrammetric Computer Vision and Image AnalysisCommission III, 2010

[3] H. Chaabouni, I. Rodes, and P. Reinartz, "Towards automatic 3-D change detection through multi-spectral and digital elevation model information fusion,' International Journal of Image and Data Fusion, 2013.

[4] P. dAngelo and P. Reinartz, "Semi-global matching results on the isprs stereo matching benchmark," in Int. Archives Photogrammetry, Remote Sensing and Spatial Information Sciences, vol. XXXVIII-4/W19, 2011.

[5] G. R. Dini, K. Jacobsen, F. Rottensteiner, M. Rajhi, and C. Heipke, " $3 \mathrm{~d}$ building change detection using high resolution stereo images and a gis database," in Int. Archives Photogrammetry, Remote Sensing and Spatial Information Sciences - XXII ISPRS Congress, vol. XXXIX-B7, 2012.

[6] C. Harris and M. Stephens, "A combined corner and edge detector," in Proceedings of the Fourth Alvey Vision Conference, 1988, pp. 147-151.

[7] M. İlsever and C. Ünsalan, Two Dimensional Change Detection Methods: Remote Sensing Applications. Springer, 2012.

[8] F. Jung, "Detecting building changes from multitemporal aerial stereoparis," ISPRS Journal of Photogrammetry and Remote Sensing, pp. 187-201, 2004.

[9] H. Murakami, K. Nakagawa, H. Hasegawa, T. Shibata, and E. Iwanami, "Change detection of buildings using an airborne laser scanner," ISPRS Journal of Photogrammetry and Remote Sensing, vol. 54, pp. 148-152, 1999.

[10] A. H. Özcan, C. Ünsalan, and P. Reinartz, "Building detection using local features and DSM data," in Proceedings of RAST'13, 2013, pp. 139-143.

[11] B. Sirmacek and C. Ünsalan, "A probabilistic framework to detect buildings in aerial and satellite images," IEEE Transactions on Geoscience and Remote Sensing, vol. 49, no. 1, pp. 211-221, 2011.

[12] N. Sofina and M. Ehlers, "Object-based change detection using highresolution remotely sensed data and gis," in Int. Archives Photogrammetry, Remote Sensing and Spatial Information Sciences - XXII ISPRS Congress, vol. XXXIX-B7, 2012.

[13] J. Tian, S. Cui, and P. Reinartz, "Building change detection based on satellite stereo imagery and digital surface models," IEEE Transactions on Geoscience and Remote Sensing, vol. 52, no. 1, pp. 406-417, 2014.

[14] J. Tian and P. Reinartz and P. d'Angelo and M. Ehlers "Region-based automatic building and forest change detection on Cartosat-1 stereo imagery," in ISPRS Journal of Photogrammetry and Remote Sensing, 2013.

[15] X. Tong, Z. Hong, S. Liu, Z. Zhang, H. Xie, Z. Li, S. Yang, W. Wang, and F. Bao, "Building-damage detection using pre- and post-seismic high-resolution satellite stereo imagery: A case study of the may 2008 wenchuan earthquake," ISPRS Journal of Photogrammetry and Remote Sensing, pp. 13-27, 2012.

[16] M.P. Dare, "Shadow analysis in high-resolution satellite imagery of urban areas," in Photogrammetric Engineering and Remote Sensing, 2005, pp. 169177.

[17] P. Soille,"Morphological Image Analysis: Principles and Applications," Springer-Verlag, 1999, pp. 170-171.

\section{ACKNOWLEDGMENT}

This work is supported by TUBITAK under project no $110 \mathrm{E} 302$. 